\title{
A model for chaotic dielectric microresonators
}

\author{
J. P. Keating and M. Novaes \\ School of Mathematics, University of Bristol, Bristol BS8 1TW, United Kingdom \\ H. Schomerus \\ Department of Physics, Lancaster University, Lancaster LA1 4YB, United Kingdom
}

\begin{abstract}
We develop a random-matrix model of two-dimensional dielectric resonators which combines internal wave chaos with the deterministic Fresnel laws for reflection and refraction at the interfaces. The model is used to investigate the statistics of the laser threshold and line width (lifetime and Petermann factor of the resonances) when the resonator is filled with an active medium. The laser threshold decreases for increasing refractive index $n$ and is smaller for TM polarization than for TE polarization, but is almost independent of the number of out-coupling modes $N$. The Petermann factor in the line width of the longest-living resonance also decreases for increasing $n$ and scales as $\sqrt{N}$, but is less sensitive to polarization. For resonances of intermediate lifetime, the Petermann factor scales linearly with $N$. These qualitative parametric dependencies are consistent with the random-matrix theory of resonators with small openings. However, for a small refractive index where the resonators are very open, the details of the statistics become non-universal. This is demonstrated by comparison with a particular dynamical model.
\end{abstract}

\section{INTRODUCTION}

Two-dimensional dielectric microresonators attract considerable attention because of their prospective application as microlasers and single-photon cavities, as well as sensors in chemical and biological systems [1-6]. These systems also allow the study of generic properties of partially confined waves in a clean setting, and hence provide a route to acquire knowledge which can be transferred, e.g., to mesoscopic electronic devices [7]. The analogy is most complete when comparing non-interacting electrons in cooled, patterned two-dimensional semiconductor devices (which usually leak through quantum-point contacts) with passive optical (or micro-wave) resonators equipped with small openings. For such geometries, semiclassical methods and random-matrix theory have provided a deep theoretical understanding of the spectral and transport properties, which have been found to agree with numerous experiments and numerical computations [8-13]. In particular, it is now well established that the predictions of random-matrix theory, designed for wave-chaotic systems with strong mode-mixing, are of universal applicability when the width of the openings $W$ is much less than the linear system size $L$, implying a long mean lifetime in the system. For twodimensional ballistic geometries, this requirement can be quantified by comparing the numbers $N \propto W k$ of outcoupled channels at wavenumber $k$ to the number of channels $M \propto L k$ which are mixed by the scattering at the boundaries. Standard random-matrix universality then requires $M \gg N$.

Dielectric microresonators, however, leak everywhere around the interface and are far more open than the resonators considered in standard random-matrix theory. The photonic confinement relies on internal reflection, which only becomes perfect for angles of incidence above a critical value. As a consequence, the effective 'open- ness' increases with the system size, resulting in $N \propto M$. Universality is no longer guaranteed, and a more detailed modelling is required. This modelling also has to account for the possible presence of an active medium, essential for microlasers, which have been manufactured in many forms and materials [4, 14-27].

In this paper we develop a quantum-dynamical description of two-dimensional dielectric microresonators which combines wave-chaotic propagation of photons inside the resonator with the Fresnel laws for reflection and refraction at the interface. The general construction is based on a variant of the quantum surface-of-section method [2833]. The propagation inside the resonator is expressed in terms of an internal scattering matrix, which we specify by either using random-matrix theory [10, 34] or a quantum-dynamical paradigm of chaotic wave propagation, the quantum kicked rotator [35]. Similar models have recently been developed for mesoscopic electronic and hybrid-superconducting devices [36-40], which require different boundary conditions to open up the system and do not allow for amplification.

The random-matrix and quantum kicked rotator variants of the model are explored to investigate the threshold and the quantum-limited line width of wave-chaotic dielectric microlasers. The laser threshold is related to the decay rate $\Gamma$ of the longest living resonances, and can be read off from the imaginary part of the frequency (which is complex for the non-hermitian operator describing an open system). For a good, conventional laser resonator (with almost-perfect mirrors), the line width (full width at half maximum of the Lorentzian line shape) is given by the Shawlow-Townes formula [41]

$$
\Delta \omega_{S T}=\frac{1}{2} \frac{\Gamma^{2}}{I}
$$

where $I$ is the total output intensity. In an open resonator, the line width is enhanced with respect to this 
prediction by the so-called Petermann factor $K$,

$$
\Delta \omega=K \Delta \omega_{S T},
$$

which can be related to the mutual non-orthogonality of the resonance modes obtained from the non-hermitian operator [42-47], and has been studied extensively in a wide range of quantum-optical frameworks [48-59]. We investigate how $\Gamma$ and $K$ depend on the refractive index $n$ and the size of the resonator (quantified by $N$ and $M$ ), and also discriminate between the two possible polarizations TE and TM (where the electric or magnetic field lies inside the resonator plane, respectively). The results are contrasted with predictions of random-matrix theory for resonators with small openings $[12,44-46,60,61]$.

We start this paper in Section II with a brief summary of the basic concepts involved in the description of twodimensional dielectric microresonators. The quantumdynamical models are formulated in Section III. In Section IV we present the statistics of decay rates $\Gamma$ (determining the laser threshold) and Petermann factors $K$ (determining the line width). These results are obtained by numerical sampling of the random-matrix ensemble and the parameter space of the quantum-kicked rotator. A summary of the results and conclusions is presented in Section V.

\section{TWO-DIMENSIONAL DIELECTRIC RESONATORS}

We consider planar dielectric microresonators of a small width $\Delta z$ whose material properties are characterized by a refractive index $n(\omega)$, which may depend on the angular frequency $\omega$. Amplification is modelled within an effective medium approach, so that the refractive index is complex, with $-\operatorname{Im} n>0$ proportional to the amplification rate. The refractive index is taken as homogeneous within the resonator and unity in the surrounding medium. The geometry of the resonator is specified by a region $D$ in the $(x, y)$ plane.

We assume that only the lowest-lying transverse mode is excited, which provides the best confinement at the planar interfaces and hence results in the longest-living resonances. For small wavelengths $(\lambda=c / \omega \ll L$, where $c$ is the velocity of light and $L$ is the linear dimension of the resonator in the plane), the radiation outside the resonator is mostly confined to the resonator plane. Throughout this plane, the electromagnetic field can then be described by a scalar wavefunction $\psi(\mathbf{r}), \mathbf{r}=(x, y)$, which represents the electric or magnetic field component parallel to the $z$ axis (TM or TE polarization, respectively).

The wavefunction $\psi(x, y)$ obeys the Helmholtz equation

$$
\left[c^{2} \nabla^{2}+n^{2}(\mathbf{r}) \omega^{2}\right] \psi(\mathbf{r})=0
$$

At the interfaces the wavefunction $\psi$ and its normal derivative $\partial_{\perp} \psi$ are continuous for TM polarization, while for TE polarization these continuity requirements are fulfilled by $\psi$ and $n^{-2} \partial_{\perp} \psi$.

For interfaces with no curvature, these boundary conditions result in Snell's law

$$
\sin \eta=n \sin \chi
$$

which relates the angle of incidence $\eta$ from a wave approaching the interface to the angle of refraction $\chi$ of the wave within the refractive medium. The reflection probabilities for both polarizations are given by the Fresnel laws $[62,63]$

$$
R_{T M}=\frac{\sin ^{2}(\chi-\eta)}{\sin ^{2}(\chi+\eta)}, \quad R_{T E}=\frac{\tan ^{2}(\chi-\eta)}{\tan ^{2}(\chi+\eta)} .
$$

From within the medium, total reflection occurs for angles of incidence $\chi>\chi_{c}$ larger than the critical angle, which is determined by the condition $\sin \chi_{c}=1 / n$.

For the more complicated interface geometry of a microresonator, the radiative properties are encoded in the scattering matrix $S(\omega)$, which relates the incoming and outgoing amplitudes $\psi_{i}^{\text {(out) }}=\sum_{j} S_{i j} \psi_{j}^{(\text {(in) }}$ in a suitably chosen, flux-normalized basis of scattering states. The poles of the scattering matrix are defined by the condition $S\left(\omega_{m}\right)=\infty$. For $\omega=\omega_{m}$, the Helmholtz equation (3) permits solutions $\psi_{m}(\mathbf{r})$ with purely outgoing boundary conditions, $\psi_{j}^{(\mathrm{in})}=0$. For a real refractive index, these poles lie in the lower part of the complex plane $\operatorname{Im} \omega_{m} \equiv-\Gamma_{m} / 2<0$, where $\Gamma_{m}$ is the cold-cavity decay rate of the mode. Amplification shifts the poles towards the real axis. Within this effective-medium approach, the laser threshold is reached when the first pole crosses the real axis, so that the purely outgoing wave field of the laser can be realized at physical, real frequencies $\omega=\omega_{m}$ $[64,65]$.

The active medium within the resonator emits radiation due to the spontaneous emission of photons and their subsequent amplification. Assuming total population inversion of the active transition within the medium, the frequency-resolved output intensity is given by $[66,67]$

$$
I(\omega)=\frac{1}{2 \pi} \operatorname{tr}\left[S^{\dagger}(\omega) S(\omega)-1\right] .
$$

This expression vanishes for a passive medium (with a real refractive index), for which the scattering matrix is unitary.

Close to the laser threshold, the radiation is dominated by a Lorentzian peak around $\omega=\operatorname{Re} \omega_{m}$. The width of the peak can be associated to the spontaneous emission processes, which perturb the amplitude and phase of the emitted radiation. The Shawlow-Townes formula (1) arises when the resonance in the intensity (6) is calculated within Breit-Wigner perturbation theory, which is based on the mutually orthogonal modes of the closed resonator. Equation (2) including the Petermann factor accounts for the non-orthogonality of the modes in the open resonator. For $\omega L \gg c$, the Petermann factor can 
be expressed in terms of the resonance wave function as $[43,68]$

$$
K_{m}=\frac{\left.\left.\left|\int_{D} d \mathbf{r}\right| \psi_{m}(\mathbf{r})\right|^{2}\right|^{2}}{\left|\int_{D} d \mathbf{r} \psi_{m}(\mathbf{r})^{2}\right|^{2}} .
$$

Above the laser threshold, the resonator becomes unstable, since the gain in the medium outweighs the losses through the interfaces. The steady-state intensity is limited by pumping and saturation of the medium, which requires study of the non-linear regime as done, e.g., in Refs. [51, 56, 69]. The feedback with the medium stabilizes the amplitude of the laser, but not the phase, whose dynamics gives rise to a finite laser line width. The resulting width is half the width obtained from the cold-cavity calculation, which also includes the amplitude fluctuations [48]. Equations (1) and (2) include this reduction factor of one-half.

\section{QUANTUM-DYNAMICAL MODEL}

\section{A. Construction}

In order to set up the quantum-dynamical model of a dielectric microresonator we separate the motion within the cavity, which is assumed to be wave-chaotic, from the reflection and refraction processes at the resonator interfaces, which is assumed to be governed by the Snell and Fresnel laws (4) and (5), respectively.

The construction follows a variant of the quantum surface-of-section method [28-32] which we adapt to the specifics of wave propagation in a microresonator, and bares similarities to the methods used in an efficient numerical scheme for specific cavities [33]. For motivation let us first consider the classical ray dynamics in the system. The successive internal reflections of these rays at the interfaces are conveniently represented in terms of Birkhoff's canonical coordinates, given by the length along the boundary, $q$, and the sine of the angle of incidence, $p=\sin \chi$. The internal ray dynamics is then reduced to a sequence of points $\left(q_{n}, p_{n}\right)$, which are generated by an area-preserving map $\mathcal{M}:\left(q_{n}, p_{n}\right) \rightarrow$ $\left(q_{n+1}, p_{n+1}\right)$. At each encounter with the interface, a ray is split into a refracted part, which escapes to the exterior, and a reflected part which remains inside the resonator. The relative amount of reflection is measured by the reflection coefficients (5), which in Birkhoff coordinates are written

$$
\begin{aligned}
& R_{T M}(p)=\left(\frac{\sqrt{1-p^{2}}-\sqrt{n^{-2}-p^{2}}}{\sqrt{1-p^{2}}+\sqrt{n^{-2}-p^{2}}}\right)^{2}, \\
& R_{T E}(p)=\left(\frac{\sqrt{1-p^{2}}-n \sqrt{1-n^{2} p^{2}}}{\sqrt{1-p^{2}}+n \sqrt{1-n^{2} p^{2}}}\right)^{2}
\end{aligned}
$$

for $|p|<1 / n$, while $R=1$ for $|p|>1 / n$.
In wave optics, a similar separation of the internal dynamics and the encounters with the interface can be carried out for $k L \gg 1$. In this limit, the internal wave function $\psi^{(\mathrm{int})}=\psi^{(\mathrm{int}, \mathrm{in})}+\psi^{(\mathrm{int}, \text { out })}$ can be separated into the component $\psi^{\text {(int,in) }}$ which propagates away from the interfaces, towards the interior of the resonator, and the component $\psi^{\text {(int,out) }}$ which propagates towards the interfaces. Because the separation relies on the propagation direction it is best carried out in momentum space, corresponding to states with a well defined canonical coordinate $p$. The geometry of the collision with the interface is encoded in the internal scattering matrix $F(\omega)$, which relates the components of the internal wave function,

$$
\psi^{(\text {int,out })}=F(\omega) \psi^{(\text {int,in })} .
$$

This is equivalent to Bogomolny's transfer operator [29], originally introduced for closed systems. The matrix $F(\omega)$ is unitary and symmetric, the latter property arising from the time-reversal symmetry in a dielectric medium. The dimension $M \sim \operatorname{Int}[C n \omega / \pi c]$ of $F(\omega)$ depends on the perimeter $C$ of the interface, which is proportional to the system size, $C \propto L$.

In the classical limit $\omega L / c \propto M \rightarrow \infty, F(\omega)$ corresponds to the map $\mathcal{M}$. When $\omega L / c \gg 1$, the momentum $p$ is quasi-continuous,

$$
p_{l} \sim \frac{2 l-M-1}{2 M}, \quad l=1, \ldots, M .
$$

At the interfaces the internal wave function is coupled to the external wave field, which can be decomposed in the conventional scattering states $\psi^{(\text {ext })}=\psi^{(\text {ext,in) }}+$ $\psi^{\text {(ext,out) }}$. The coupling is of the general form

$$
\begin{aligned}
\psi^{(\text {int,in })} & =\mathcal{R} \psi^{(\text {int,out })}+\mathcal{T} \psi^{(\text {ext,in })}, \\
\psi^{(\text {ext,out })} & =-\mathcal{R} \psi^{(\text {ext,in })}+\mathcal{T} \psi^{(\text {int,out })},
\end{aligned}
$$

where $\mathcal{R}$ is the reflection matrix and $\mathcal{T}$ is the transmission matrix, constrained by $\mathcal{R}^{\dagger} \mathcal{R}+\mathcal{T}^{\dagger} \mathcal{T}=1$. The linear Eqs. (9) and (11) can be solved to arrive at the scattering matrix

$$
S(\omega)=-\mathcal{R}+\mathcal{T} F(\omega) \frac{1}{1-\mathcal{R} F(\omega)} \mathcal{T} .
$$

This general form of the scattering matrix has also been encountered in recent investigations of electronic transport and superconducting hybrid structures with small ballistic openings [36-40].

Our construction of the quantum-dynamical model is completed by concrete specifications of the matrices $\mathcal{R}$ and $F(\omega)$. For $k L \gg 1$, Snell's law dictates that the matrices $\mathcal{R}$ and $\mathcal{T}$ in Eq. (11) become diagonal between states which conserve $n p$, while the Fresnel laws (8) deliver the values of the diagonal elements,

$$
\mathcal{R}_{l m}=\delta_{l m} \sqrt{R\left(p_{l}\right)}, \quad \mathcal{T}_{l m}=\delta_{l m} \sqrt{1-R\left(p_{l}\right)},
$$

with $p_{l}$ given by Eq. (10). 
Concerning the internal dynamics encoded in $F(\omega)$, we now follow two routes - we either employ randommatrix theory or a generic wave-chaotic quantum map, the quantum kicked rotator.

\section{Random-matrix model}

It is commonly accepted that statistical properties of wave systems with chaotic ray dynamics are well described by random-matrix theory. In the present case of a dielectric system, which preserves time-reversal symmetry, this approach amounts to assuming that $F$ at fixed, real $\omega$ can be represented by a unitary symmetric matrix randomly drawn from the Circular Orthogonal Ensemble (COE) [34]. This substitution should best capture the statistical features of the longest-living resonances, since in a realistic resonator ray chaos is only established after a few internal reflections.

In general, the frequency dependence of $F(\omega)$ is complicated. In the subsequent analysis we will only require the local dependence around the poles of the scattering matrix, and employ as a simple approximation

$$
F(\omega)=\exp (i n \omega \bar{L} / c) U, \quad U \text { from } \operatorname{COE}(M), \text { fixed, }
$$

where $\bar{L}$ is the mean propagation distance between two reflections. Sabine's law of room acoustics delivers the universal expression $\bar{L}=\pi A / C$, where $A$ is the area of the resonator and $C$ is its perimeter. Our approximation entails $(2 \pi)^{-1} \operatorname{Im} \operatorname{tr} F^{\dagger} \frac{d F}{d \omega}=M n \bar{L} / 2 c \pi \sim A n \omega / 2 \pi c$. This reproduces the correct expression for the mean density of states, while a more elaborate modelling of the $\omega$ dependence would also describe fluctuations around this value.

The random-matrix model of two-dimensional wavechaotic microresonators is obtained by combining Eqs. (12), (13), and (14). In order to simplify the notation we introduce the quasi-energy

$$
\theta=n \omega \bar{L} / c
$$

This delivers the scattering matrix

$$
S=-\mathcal{R}+\mathcal{T} U \frac{1}{\exp (-i \theta)-\mathcal{R} U} \mathcal{T}
$$

where $U$ is an $M \times M$ matrix from the $\mathrm{COE}$, while the diagonal matrix $\mathcal{R}$ is given in Eq. (13).

\section{Quantum kicked rotator model}

The quantum kicked rotator [35] is a quantized version of the classical standard map on the torus $(q, p) \in[0,1)^{2}$, which consists of a combination of torsions $p \rightarrow p+q$ and nonlinear 'kicks' $q \rightarrow q+f(p)$, where $f(p)=f(p+1)=$ $-d v / d p$ is periodic and can be represented as the derivative of a potential $v(p)$. In order to break all symmetries apart from time-reversal symmetry we employ a quantum kicked rotator corresponding to a torsion followed by a kick with

$$
\begin{aligned}
& f(p)=K_{1} \sin (2 \pi p)+K_{2} \sin (4 \pi p+\alpha), \\
& v(p)=\frac{K_{1}}{2 \pi} \cos (2 \pi p)+\frac{K_{2}}{4 \pi} \cos (4 \pi p+\alpha),
\end{aligned}
$$

where the kicking strengths $K_{1}, K_{2}$ and the shift $\alpha$ are free parameters. The classical dynamics is known to be chaotic for $K_{1}, K_{2} \gtrsim 8$.

The propagator, or Floquet matrix, takes the form

$$
U_{n m}=\frac{1}{\sqrt{i M}} e^{\frac{i \pi}{M}(m-n)^{2}-\frac{i M}{2}\left[v\left(\frac{n}{M}\right)+v\left(\frac{m}{M}\right)\right]} .
$$

The quantum kicked rotator model of a wave-chaotic microresonator follows when this matrix is introduced into the scattering matrix (16).

\section{B. Resonances and the Petermann factor}

We now explore the general structure of the scattering matrix in the quantum-dynamical model and derive general relations for the resonances and the Petermann factor.

The scattering matrix (12) diverges when the resonant denominator $1-\mathcal{R} F(\omega)$ has a vanishing eigenvalue. The matrix $\mathcal{R} F(\omega)$ describes one round trip of a wave which propagates through the cavity, and then is internally reflected at the interface. This quantization condition is analogous to the scattering-matrix quantization condition in a closed systems $[70,71]$, which is recovered for $\mathcal{R}=1$.

For the specific form (16) of the scattering matrix, the quantization condition takes the form

$$
\mathcal{R} U \Psi_{m}=\exp \left(-i \theta_{m}\right) \Psi_{m}
$$

which is of the form of an eigenvalue equation with eigenvalue $\exp \left(-i \theta_{m}\right)$. The matrix $\mathcal{R} U$ can be interpreted as a reduced round-trip operator. Since $\mathcal{R} U$ is subunitary, $\mathcal{R} U(\mathcal{R} U)^{\dagger}=\mathcal{R} \mathcal{R}^{\dagger}<1$, all eigenvalues are submodular, $\left|\exp \left(-i \theta_{m}\right)\right|<1$; therefore the quasienergies $\theta_{m}$ have a negative imaginary part, $\gamma_{m} \equiv-2 \operatorname{Im} \theta_{m}>0$.

The subunitarity of $\mathcal{R} U$ furthermore implies that the right eigenvectors $\Psi_{m}$ defined by Eq. (20) are not mutually orthogonal. However, they form a bi-orthogonal set with the left eigenvectors,

$$
\left\langle\Phi_{l} \mid \Psi_{m}\right\rangle=\delta_{l m}
$$

where the latter are defined by the adjoint eigenvalue problem

$$
\Phi_{m}^{\dagger} \mathcal{R} U=\Phi_{m}^{\dagger} \exp \left(-i \theta_{m}\right)
$$

In the original variables, the eigenphases $\theta_{m}$ can be interpreted in two different ways: (1) They are poles 
$\theta_{m}=n \omega_{m} \bar{L} / c$ of the scattering matrix for fixed (possibly complex) refractive index $n$ but variable, generally complex $\omega_{m}$, (2) they deliver the threshold condition $\theta_{m}=n_{m} \omega_{m}^{\prime} \bar{L} / c$ of a given resonant state for real $\omega_{m}^{\prime}$ and a refractive index $n_{m}=n^{\prime}+i n_{m}^{\prime \prime}$ for which only the real part is fixed. For the scattering matrix (16), both problems are intimately related since only the product $n \omega$ enters the expressions. The cold-cavity poles $\omega_{m}^{\text {(cold) }}$ are obtained for a real refractive index $n=n^{\prime}$. The threshold amplification is then given by

$$
n_{m}^{\prime \prime}=n^{\prime} \operatorname{Im} \omega_{m}^{(\text {cold })} / \operatorname{Re} \omega_{m}^{(\text {cold })}
$$

For realistic dielectric microresonators, this relation is indeed well established in the semiclassical limit $L \gg \lambda=$ $\operatorname{Re} \omega / c$ [72]. In such systems, it is moreover reasonable to assume that the width of the amplification window of the active medium is much less than the center of this window, $\operatorname{Re} \omega_{m}$. Within the amplification window, the threshold values $n_{m}^{\prime \prime}$ then mainly depend on the imaginary part $\operatorname{Im} \omega_{m}^{(\text {cold })} \equiv-\Gamma_{m} / 2$, where $\Gamma_{m}$ is the coldcavity decay rate. The laser threshold is hence determined by the longest-living resonances of the cold cavity, which are characterized by a small value of $\Gamma_{m}$.

We now turn to the radiation emitted close to resonance, brought about by steering the amplification close to the threshold value (23), $n^{\prime \prime}=n_{m}^{\prime \prime}+\delta n$, while keeping $\omega=\operatorname{Re} \omega_{m}^{\text {(cold) }}+\delta \omega$ real. We combine both deviations into the quasienergy deviation $\theta \approx \theta_{m}+\delta \theta$. The scattering matrix (16) can then be evaluated by only keeping the resonant term in the denominator,

$$
S \approx \mathcal{T} U \Psi_{m} \frac{e^{i \theta_{m}}}{e^{-i \delta \theta}-1} \Phi_{m}^{\dagger} \mathcal{T}
$$

The frequency-resolved output intensity Eq. (6) takes the form

$$
I(\theta) \approx \frac{1}{2 \pi c} K \frac{4 \sinh ^{2}\left(\operatorname{Im} \theta_{m}\right)}{|\exp (i \operatorname{Re} \delta \theta)-\exp (\operatorname{Im} \delta \theta)|^{2}},
$$

where

$$
K=\left(\Psi_{m}^{\dagger} \Psi_{m}\right)\left(\Phi_{m}^{\dagger} \Phi_{m}\right)
$$

We now can linearize in $\delta \theta$, which produces the Lorentzian lineshape

$$
I(\theta) \approx \frac{1}{2 \pi} K \frac{4 \sinh ^{2}\left(\operatorname{Im} \theta_{m}\right)}{\operatorname{Re} \delta \theta^{2}+\operatorname{Im} \delta \theta^{2}} .
$$

Since we are mainly concerned with long living resonances in a large cavity, $-\operatorname{Im} \omega_{m}=\Gamma_{m} / 2 \ll \operatorname{Re} \omega$, we further linearize the numerator and obtain in the frequency domain

$$
I(\omega) \approx \frac{1}{2 \pi} K \frac{\Gamma_{m}^{2}}{\left(\omega-\operatorname{Re} \omega_{m}^{(\text {cold })}\right)^{2}+\Delta \omega^{2} / 4},
$$

where the full width at half maximum is $\Delta \omega=$ $2 \delta n \operatorname{Re} \omega_{m}^{\text {(cold) }} / n^{\prime}$. The total intensity is given by

$$
I=\int d \omega I(\omega)=K \frac{\Gamma_{m}^{2}}{\Delta \omega} .
$$

This recovers relation (2) [excluding the factor of $1 / 2$ due to the suppression of amplitude fluctuations by the nonlinear feedback in the lasing regime]. Consequently, $K$ defined in Eq. (26) has to be identified with the Petermann factor.

Expression (26) assumes that the left and right eigenvectors are normalized according to the bi-orthogonality condition (21). When this condition is dropped, the Petermann factor takes the more general form

$$
K=\frac{\left(\Psi_{m}^{\dagger} \Psi_{m}\right)\left(\Phi_{m}^{\dagger} \Phi_{m}\right)}{\left|\Phi_{m}^{\dagger} \Psi_{m}\right|^{2}}
$$

In the present, time-reversal-symmetric situation where $U=U^{T}$, the right and left eigenvectors defined by Eqs. (20) and (22) can be chosen (via a suitable normalization) such that $\Phi=U^{*} \Psi^{*}$. This relation can be further exploited by decomposing $U=V V^{T}$ in terms of a unitary matrix $V$, which is fixed up to transformations $V \rightarrow V O$ with an arbitrary orthogonal matrix $O$. This decomposition allows us to pass to the symmetrized eigenvalue problem

$$
\begin{aligned}
& V^{T} \mathcal{R} V \tilde{\Psi}_{m}=\exp \left(-i \theta_{m}\right) \tilde{\Psi}_{m}, \\
& \tilde{\Phi}_{m}^{\dagger} V^{T} \mathcal{R} V=\exp \left(-i \theta_{m}\right) \tilde{\Phi}_{m}^{\dagger},
\end{aligned}
$$

which is solved by the right and left eigenvectors $\tilde{\Psi}=$ $V^{T} \Psi$ and $\tilde{\Phi}=V^{T} \Phi=\tilde{\Psi}^{*}$, respectively. In terms of the symmetrized eigenvectors, the Petermann factor (30) takes the form

$$
K=\frac{\left|\tilde{\Psi}_{m}^{\dagger} \tilde{\Psi}_{m}\right|^{2}}{\left|\tilde{\Psi}_{m}^{T} \tilde{\Psi}_{m}\right|^{2}}
$$

This expression is formally analogous to expression (7) for the Petermann factor in terms of the resonance wave function inside the cavity, but involves the eigenvectors $\tilde{\Psi}_{m}$ of the symmetrized reduced round-trip operator $V^{T} \mathcal{R} V$. Furthermore, Eq. (32) is formally equivalent to the expression used in earlier random-matrix theories for cavities with small openings [44-46, 61, 73-76].

\section{NUMERICAL RESULTS}

We now employ the quantum-dynamical model with scattering matrix (16) in order to investigate the statistical properties of wave-chaotic dielectric microlasers. For fixed values of the refractive index $n$ and polarization (TE or TM), the matrix $U$ is either chosen as a random representative from the $\mathrm{COE}$ or as the quantum kicked rotator (19). The laser threshold follows from Eq. (23), while the Petermann factor follows from Eq. 

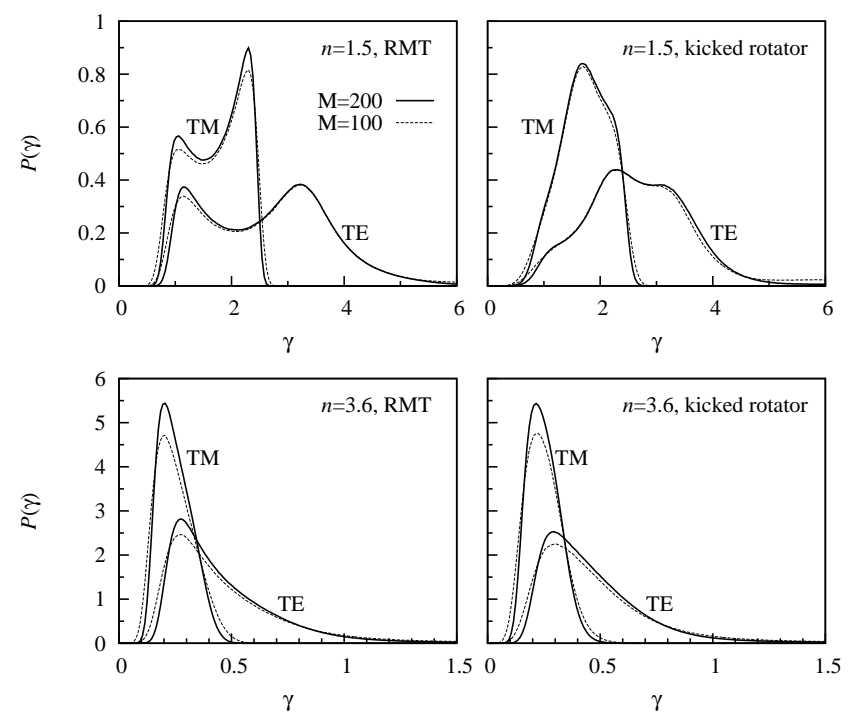

FIG. 1: Probability distribution $P(\gamma)$ of the scaled decay rate $\gamma_{m}=-2 \operatorname{Im} \theta_{m}$, obtained from the eigenvalue equation (20). In the upper panels the refractive index is $n=1.5$ while in the lower panels $n=3.6$. The left panels are computed in random-matrix theory, while the right panels are computed in the quantum kicked rotator model. The matrix dimension is $M=200$ (solid curves) and $M=100$ (dashed curves). The labels TM and TE discriminate the different polarizations. Each curve is based on $10^{5}$ realizations of $U$.

(32). In order to present the results for the life times and laser threshold we use the scaled imaginary part $\gamma_{m}=-2 \operatorname{Im} \theta_{m}=\Gamma_{m} \bar{L} / c$.

In the following we contrast the case of a relatively open resonator with refractive index $n=1.5$ (close to the value of glass) to a relatively closed resonator with $n=3.6$ (close to the value of $\mathrm{Ga}(\mathrm{Al}) \mathrm{As}$ ). In the randommatrix model, the computations are based on $10^{5}$ realizations of $U$ from the COE with matrix dimension $M=100$ or $M=200$ [77]. In the kicked-rotator model, the same number of matrices with identical dimensions are obtained by varying the parameters $\alpha, K_{1}$ and $K_{2}$. We also present $n$-dependent averages, which are based on $10^{4}$ realizations of $U$ in each of the models.

Figure 1 shows the probability distribution function $P(\gamma)$ of scaled decay rates for both polarizations and the two representative values of the refraction index. The results obtained for matrices of dimension $M=100$ and 200 are very close, suggesting a mild dependence of $P(\gamma)$ on the wavelength when the resonator size increases. For the relatively closed resonator, $n=3.6$, the distributions found in both models are almost identical. For the relatively open resonator with $n=1.5$, however, the limiting distribution in the random-matrix model is distinctly different from the result in the quantum kicked rotator model. This demonstrates that universality starts to break down as the system becomes more open, due to the greater influence of short-time dynamics (this breakdown has been studied in more detail for the spectrum and res-
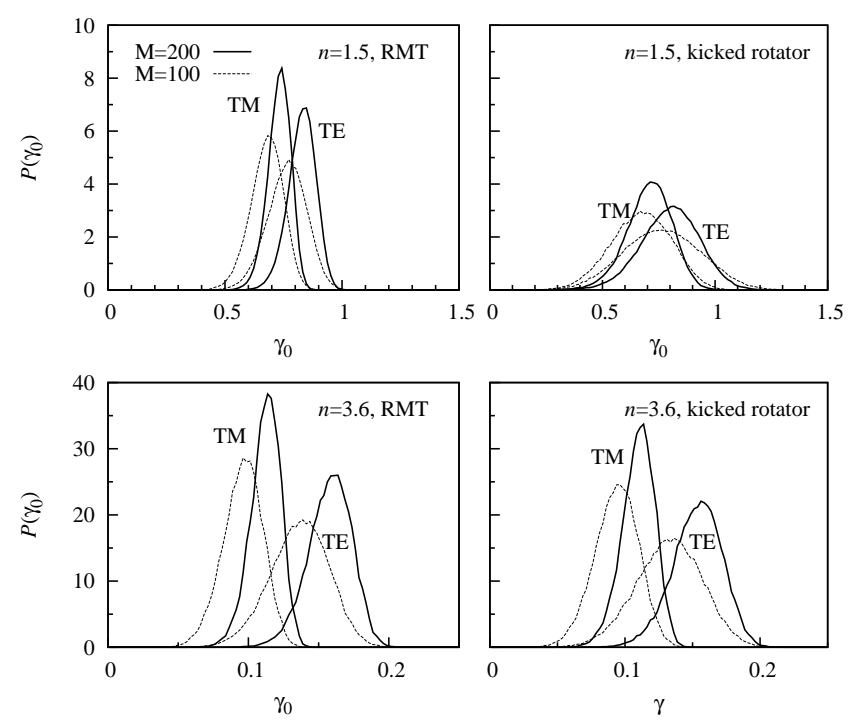

FIG. 2: Probability distribution $P\left(\gamma_{0}\right)$ of the extremal (smallest) scaled decay rate $\gamma_{0}$. The symbols and parameters are the same as in Fig. 1.

onance wave functions of ballistic systems in [40, 78, 79], using semiclassical arguments). On the other hand, the characteristic qualitative features of the distributions display a robust parameter dependence. For both values of the refractive index $n$ and in both models, the TE polarization leads to larger decay rates compared to TM. For a given polarization, the decay rates decrease with increasing $n$. Both trends are consistent with the general features of the Fresnel laws (5), which provide better confinement for TM polarization, and for large refractive indices.

For each realization, the laser threshold is determined by the extremal resonance with the smallest rescaled decay rate, which we denote by $\gamma_{0}$. Figure 2 shows the distribution $P\left(\gamma_{0}\right)$ of the extremal decay rate for the same parameters as used in Fig. 1. Again the results from both models coincide for the relatively closed resonator, while for the relatively open resonator the random-matrix model has a distinctively more narrow distribution than the quantum kicked rotator model. In all cases, TM polarization yields smaller decay rates than TE polarization, which is inherited from the behavior of $P(\gamma)$ in Fig. 1. The results also show a trend to larger decay rates as the dimension $M$ is increased. This trend can be explained by the (hardly visible) sharpening of the small- $\gamma$ flank in the distribution function $P(\gamma)$ [Fig. 1] when $M$ is increased, which suppresses the tail with very small decay rates. Such a sharpening has also been demonstrated in the random-matrix theory of cavities with small ballistic openings, where the distribution function eventually becomes discontinuous $[12,60,80,81]$.

Figure 3 shows the average value $\left\langle\gamma_{0}\right\rangle$ of the extremal decay rate as a function of the refractive index $n$. For this quantity, the results from the random-matrix and 


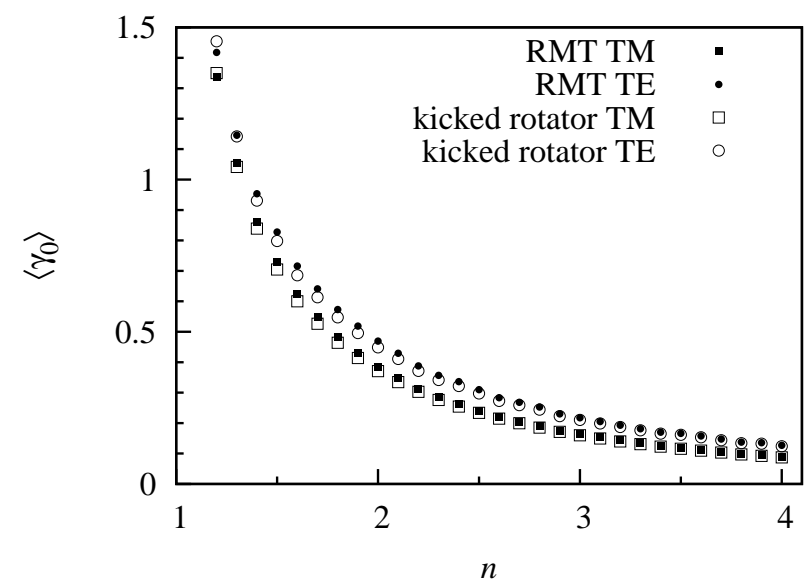

FIG. 3: Ensemble average $\left\langle\gamma_{0}\right\rangle$ of the scaled extremal decay rate $\gamma_{0}$ as a function of refractive index $n$, for TM and TE polarization (squares and circles, respectively). Solid symbols are obtained in random-matrix theory, while open symbols are obtained in the quantum kicked rotator model. Each data point is based on $10^{4}$ realizations of $U$ with matrix dimension $M=200$.

kicked-rotator models agree very well. As expected from the increasing confinement, $\left\langle\gamma_{0}\right\rangle$ decreases with increasing refractive index $n$, and is smaller for TM polarization than for TE polarization.

We now turn to the statistics of the Petermann factor. Following the results from other variants of randommatrix theory $[44-46,61,73-76]$ it has to be expected that the Petermann factor increases with increasing decay rate of a resonance, and moreover increases with increasing number of outcoupling channels. In the present class of systems this number is simply proportional to $M$.

Anticipating these trends, we show in Fig. 4 the distribution $P(\kappa)$ of rescaled Petermann factors $\kappa=K / M$. The distribution represents the Petermann factor of all resonances, without discriminating them by width; parameters are the same as in Fig. 1. The collapse of the curves demonstrates clearly the linear scaling with $M$. Smaller Petermann factors are observed for TM polarization and/or increasing refractive index, which is consistent with the reduction of decay rates [see Fig. 1]. In contrast to the distribution of decay rates, the results from the random-matrix and kicked-rotator model agree well for both refractive indices, indicating that the Petermann factor has a more universal statistics.

Figure 5 shows the conditional probability distribution $P(\kappa \mid \gamma)$, of Petermann factors with a given value of the rescaled decay rate. We again find an approximate linear scaling with $M$. The dependence on polarization and refractive index follows the same trends as in Fig. 4. Universality is also present, since the results for both models are very similar.

The line width of the microlaser is determined by the Petermann factor of the longest-living resonance, which we denote by $K_{0}$ and refer to as the extremal Petermann
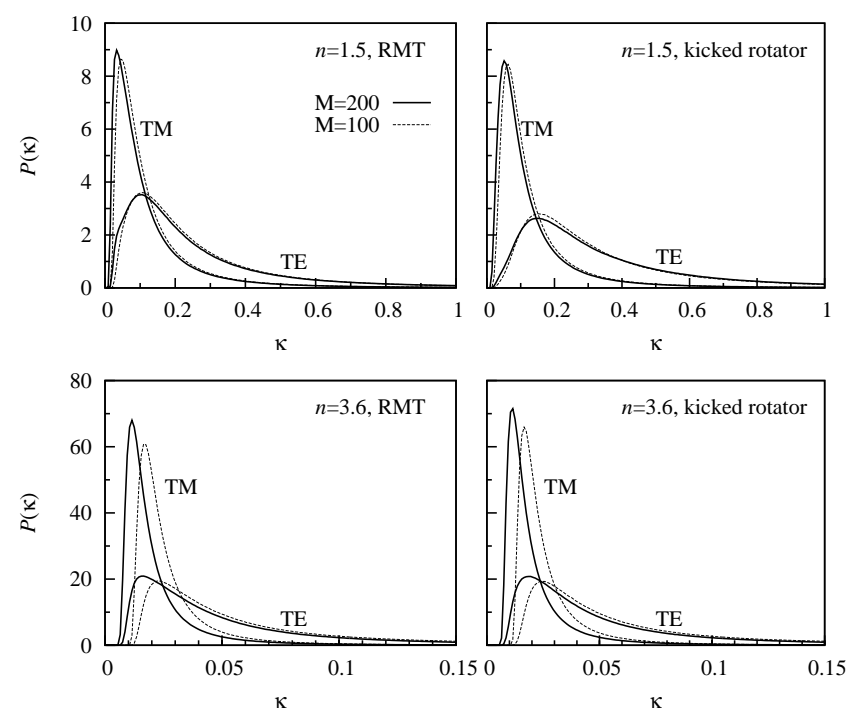

FIG. 4: Probability distribution $P(\kappa)$ of the scaled Petermann factor $\kappa=K / M$. The symbols and parameters are the same as in Fig. 1.

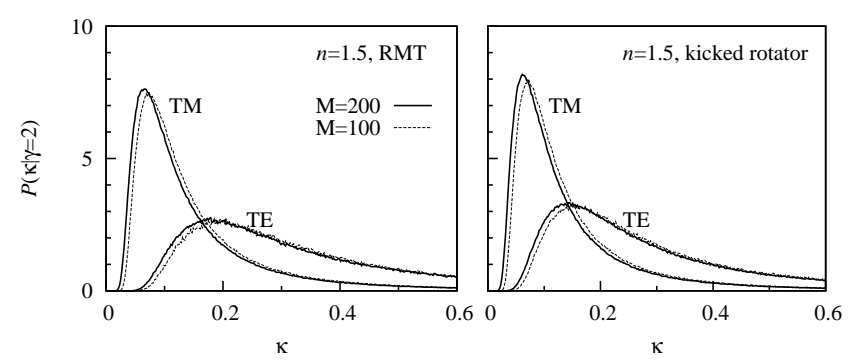

FIG. 5: Conditional probability distribution $P(\kappa \mid \gamma)$ of scaled Petermann factors $\kappa=K / M$ at a fixed value $\gamma \approx 2.0$ of the scaled decay rate. Results are shown for both polarizations and two matrix dimensions. The refractive index is $n=1.5$.

factor. (For a given realization, this is not necessarily the smallest Petermann factor among all the resonances.) As shown in Fig. 6, $K_{0}$ does not scale linearly with $M$, but scales instead with $\sqrt{M}$. This different parametric dependence can be traced to the fact that the decay rate of the extremal resonance is not fixed [Fig. 2]. The same parametric dependence, but as a function of $N$, is also obtained in the random-matrix theory of cavities with small ballistic openings, where $N \ll M[45,46]$. According to Fig. 6, the details of the statistics for the extremal Petermann factor are again non-universal for the relatively open resonator with $n=1.5$ - the distribution in the random-matrix model is more narrowly peaked than in the kicked-rotator model.

Similarly to what is observed in Fig. 4, the extremal Petermann factor in Fig. 6 is reduced when increasing refractive index $n$. However, $K_{0}$ displays a weaker polarization dependence. These trends are further underlined in Fig. 7, which shows the ensemble average $\left\langle\tilde{\kappa}_{0}\right\rangle$ of the scaled extremal Petermann factor $\tilde{\kappa}_{0}=K_{0} / \sqrt{M}$. 

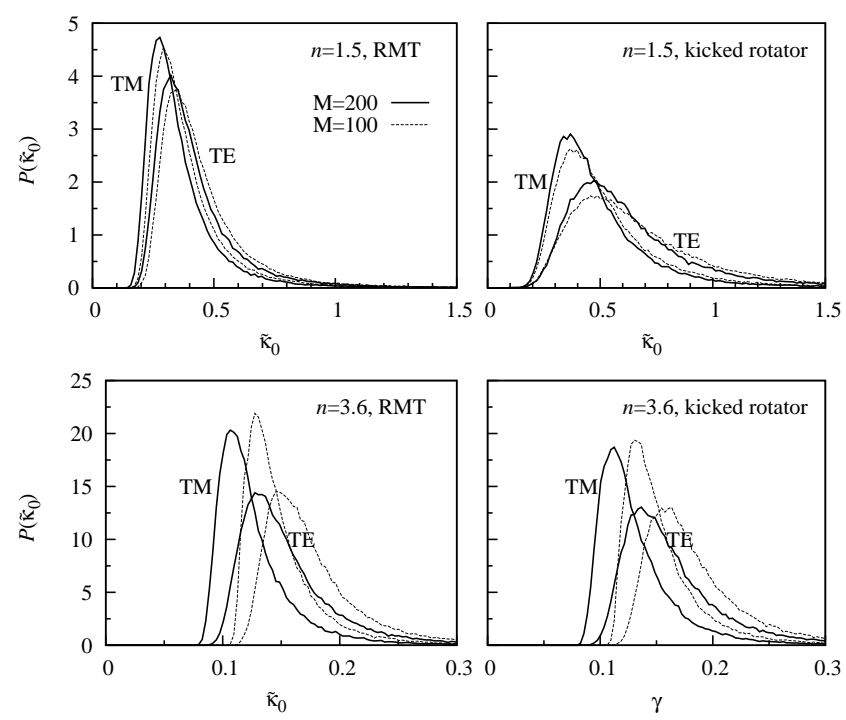

FIG. 6: (color online) Probability distribution $P\left(\tilde{\kappa}_{0}\right)$ of the scaled extremal Petermann factor $\tilde{\kappa}_{0}=K_{0} / \sqrt{M}$ of the longest-living resonance. The symbols and parameters are the same as in Fig. 1.

This average is significantly larger than the modal value, which is due to the long tail in the distribution function. The average decreases with increasing refractive index, similarly to the averaged extremal decay rate in Fig. 3. The results from the random-matrix and kicked-rotator models converge for large refractive index.

In summary, Figs. 1-3 demonstrate that the laser threshold of a wave-chaotic dielectric microresonator decreases for increasing refractive index $n$ and is smaller for TM polarization than for TE polarization. The threshold increases slowly for increasing number of out-coupling modes. Figures 6 and 7 show that the Petermann factor of a wave-chaotic dielectric microresonator decreases for increasing refractive index $n$ and scales as the squareroot of the number of out-coupling modes, but is less sensitive to polarization. Figure 4 shows that this behavior critically depends on the requirement to determine the longest-living resonance, which first reaches the laser threshold.

For all quantities, details of the statistics are modeldependent for relatively open resonators, but become universal for relatively closed resonators with a large refractive index (for very closed resonators the results eventually converge to the predictions of conventional random-matrix theory $[12,44-46,60,61])$.

\section{CONCLUSIONS}

The quantum-dynamical model of dielectric microresonators presented in this work addresses geometries which facilitate well-established wave chaos in the semiclassical limit $\lambda \ll L$, where $L$ is the typical resonator

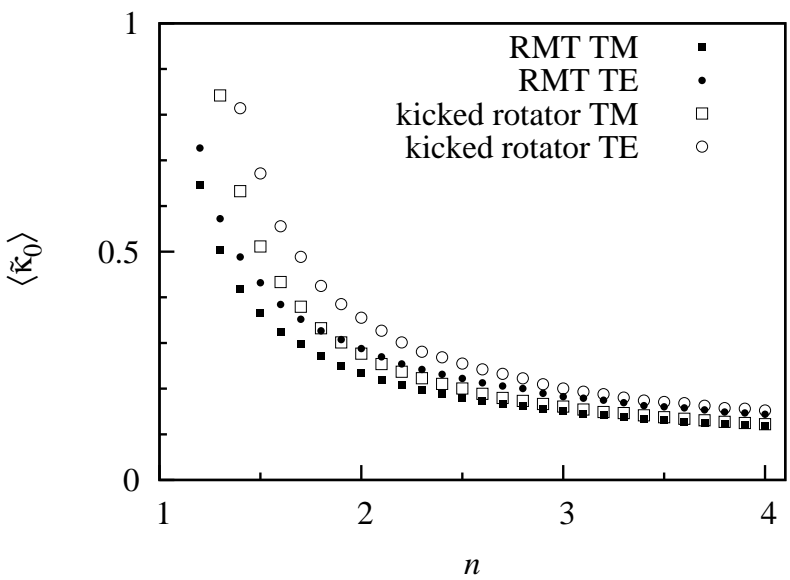

FIG. 7: Ensemble average $\left\langle\tilde{\kappa}_{0}\right\rangle$ of the scaled extremal Petermann factor $\tilde{\kappa}_{0}=K_{0} / \sqrt{M}$ as a function of refractive index $n$. The symbols and parameters are the same as in Fig. 3.

dimension and $\lambda$ is the wave length. We developed two variants of the model, one being based on random matrix theory while the other is based on the quantum-kicked rotator. In general, we found that relatively closed resonators (with a large refractive index) display universal statistics while model-dependence sets in for relatively open resonators (with a small refractive index). In the latter case the non-universal features even affect longliving resonances, which classically correspond to rays which enjoy many internal reflections at the dielectric interfaces.

The longest-living resonances determine the laser action of the resonator. We concentrated most of our efforts on the laser threshold and the quantum-limited line width, which should be directly accessible in a suitable experiment. We found that among the two competing polarizations, TM will usually win the mode competition for the most stable resonance. The threshold decreases with increasing refractive index. The Petermann factor in the line width also decreases with increasing refractive index, and furthermore scales $\propto \sqrt{L / \lambda}$.

Experimentally, Petermann factors of the laser mode have been determined for various geometries in Ref. [5759]. Recent experimental progress [82] now makes it possible to address the life time and Petermann factors of individual resonances via direct means. These methods are not restricted to the longest living resonance, and do not require an active medium (they, hence, also apply to passive or absorbing resonators). Such an experiment could serve to validate the different scaling $\propto L / \lambda$ of Petermann factors for resonances of a fixed decay rate.

Many microresonators of interest have a geometry which facilitates ray chaos only in parts of the classical phase space $[4,14-23]$. While random-matrix theory is not applicable to these systems, the kicked-rotator model can readily account for such situations when the kicking strengths are suitably reduced. In general, Eq. 
(12) for the scattering matrix provides a vehicle to study a wide range of situations, including integrable or disordered systems, by a suitable round-trip operator $\mathcal{R} U$. Equation (23) for the laser threshold and Eq. (32) for the Petermann factor do not depend on the specific choice of the round-trip operator and can serve as a starting point for further analytical considerations.

This work was supported by the EPSRC and by the European Commission, Marie Curie Excellence Grant MEXT-CT-2005-023778.
11 Focus issue Single photons on demand, edited by P. Grangier, B. Sanders, and J. Vuckovic, New J. Phys. 6 (2004).

[2] Optical Microcavities, edited by K. Vahala (World Scientific, Singapore, 2005).

[3] A. Ashkin, Optical Trapping and Manipulation of Neutral Particles Using Lasers (World Scientific, Singapore, 2001).

[4] J. U. Nöckel and R. K. Chang, in Cavity-Enhanced Spectroscopies, edited by R. D. van Zee and J. P. Looney (Academic Press, 2002), pp. 185-226.

[5] H. Cao, Waves Random Media 13, R1 (2003).

[6] E. Krioukov, D. J. W. Klunder, A. Driessen, J. Greve, and C. Otto, Opt. Lett. 27, 512 (2002).

[7] S. Datta, Electronic Transport in Mesoscopic Systems, (Cambridge University Press; Cambridge, UK, 1997).

[8] H.-J. Stöckmann, Quantum Chaos: An Introduction (Cambridge University Press, Cambridge, UK, 1999).

[9] K. Efetov, Supersymmetry in Disorder and Chaos (Cambridge University Press, Cambridge, UK, 1997).

[10] F. Haake, Quantum Signatures of Chaos, 2nd edition (Springer, Berlin, 2001).

[11] C. W. J. Beenakker, Rev. Mod. Phys. 69, 731 (1997).

[12] Y. V. Fyodorov and H.-J. Sommers, J. Math. Phys. 38, 1918 (1997).

[13] T. Guhr, A. Müller-Groeling, and H. A. Weidenmüller, Phys. Rep. 299, 189 (1998).

[14] Y. Yamamoto and R. Slusher, Phys. Today 46, 66 (1993).

[15] C. Gmachl, F. Capasso, E. E. Narimanov, J. U. Nöckel, A. D. Stone, J. Faist, D. L. Sivco, and A. Y. Cho, Science 280, 1556 (1998).

[16] U. Vietze, O. Krauss, F. Laeri, G. Ihlein, F. Schüth, B. Limburg, and M. Abraham, Phys. Rev. Lett. 81, 4628 (1998).

[17] S.-B. Lee, J.-H. Lee, J.-S. Chang, H.-J. Moon, S. W. Kim, and K. An, Phys. Rev. Lett. 88, 033903 (2002).

[18] N. B. Rex, H. E. Tureci, H. G. L. Schwefel, R. K. Chang, and A. D. Stone, Phys. Rev. Lett. 88, 094102 (2002).

[19] H. G. L. Schwefel, N. B. Rex, H. W. Tureci, R. K. Chang, and A. D. Stone, J. Opt. Soc. Am. B 21, 923 (2004).

[20] M. Kneissl, M. Teepe, N. M. Johnson, G. D. Chern, and R. K. Chang, Appl. Phys. Lett. 84, 2485 (2004).

[21] T. Ben-Messaoud and J. Zyss, Appl. Phys. Lett. 86 241110 (2004)

[22] F. Courvoisier, V. Bouton, J. P. Wolf, R. K. Chang and J. Zyss, Opt. Lett. 30, 738 (2005).

[23] S.-Y. Lee, S. Rim, J.-W. Ryu, T.-Y. Kwon, M. Choi, and C.-M. Kim, Phys. Rev. Lett. 93, 164102 (2004).

[24] T. Harayama, T. Fukushima, S. Sunada, and K. S. Ikeda, Phys. Rev. Lett. 91, 073903 (2003).

[25] T. Fukushima and T. Harayama, IEEE J. Sel. Top. Quantum Electron. 10, 1039 (2004).

[26] M. Lebental, J. S. Lauret, R. Hierle, and J. Zyss, Appl. Phys. Lett. 88, 031108 (2006).
[27] T. Tanaka, M. Hentschel, T. Fukushima and T. Harayama, Phys. Rev. Lett. 98, 033902 (2007).

[28] B. Crespi, G. Perez, and S. J. Chang, Phys. Rev. E 47, 986 (1993).

[29] E.B. Bogomolny, Nonlinearity 5, 805 (1992).

[30] B. Georgeot and R. E. Prange, Phys. Rev. Lett. 74, 4110 (1995).

[31] R.O. Vallejos and A.M. Ozorio de Almeida, Ann. Phys. 278, 86-108 (1999).

[32] A.M. Ozorio de Almeida and R.O. Vallejos, Physica E 9, 488-493 (2001)

[33] H. E. Tureci, H. G. L. Schwefel, P. Jacquod, A. D. Stone, Progress in Optics 47, 75 (2005).

[34] M. L. Mehta, Random Matrices (Academic Press, New York, 1991)

[35] F. M. Izrailev, Phys. Rep. 196, 299 (1990).

[36] Y. V. Fyodorov and H.-J. Sommers, JETP Lett. 72, 422 (2000).

[37] P. Jacquod, H. Schomerus, and C. W. J. Beenakker, Phys. Rev. Lett. 90, 207004 (2003).

[38] J. Tworzydło, A. Tajic, H. Schomerus and C.W.J. Beenakker, Phys. Rev. B 68, 115313 (2003).

[39] A. Ossipov, T. Kottos and T. Geisel, Europhys. Lett. 62, 719 (2003).

[40] H. Schomerus and P. Jacquod, J. Phys. A: Math. Gen. 38, 10663 (2005).

[41] A. L. Schawlow and C. H. Townes, Phys. Rev. 112, 1940 (1958).

[42] K. Petermann, IEEE J. Quantum Electron. 15, 566 (1979).

[43] A. E. Siegman, Phys. Rev. A 39, 1253 (1989); Phys. Rev. A 39, 1264 (1989).

[44] M. Patra, H. Schomerus, and C. W. J. Beenakker, Phys. Rev. A 61, 23810 (2000).

[45] K. M. Frahm, H. Schomerus, M. Patra, and C. W. J. Beenakker, Europhys. Lett. 49, 48 (2000).

[46] H. Schomerus, K.M. Frahm, M. Patra and C.W.J. Beenakker, Physica A 278, 469 (2000).

[47] M. V. Berry, J. Mod. Opt. 50, 63 (2003).

[48] P. Goldberg, P. W. Miloni, and B. Sundaram, Phys. Rev. A 44, 1969 (1991).

[49] M. Brunel, G. Ropars, A. Le Floch, and F. Bretenaker, Phys. Rev. A 55, 4563 (1997).

[50] P. Grangier and J.-P. Poizat, Eur. Phys. J. D 1, 97 (1998).

[51] S. M. Dutra, K. Joosten, G. Nienhuis, N. J. van Druten, A. M. van der Lee, M. P. van Exter, and J. P. Woerdman, Phys. Rev. A 59, 4699 (1999).

[52] P. J. Bardroff and S. Stenholm, Phys. Rev. A 61, 023806 (2000).

[53] M. P. van Exter, N. J. van Druten, A. M. van der Lee, S. M. Dutra, G. Nienhuis, and J. P. Woerdman, Phys. Rev. A 63, 043801 (2001)

[54] Y.-J. Cheng and A. E. Siegman, Phys. Rev. A 68, 043808 
(2003).

[55] Y.-J. Cheng, Phys. Rev. Lett. 97, 093601 (2006).

[56] G. Hackenbroich, J. Phys. A: Math. Gen. 38, 10537 (2005).

[57] Y.-J. Cheng, C. G. Fanning, and A. E. Siegman, Phys. Rev. Lett. 77, 627 (1996).

[58] M. A. van Eijkelenborg, A. M. Lindberg, M. S. Thijssen, and J. P. Woerdman, Phys. Rev. Lett. 77, 4314 (1996); Phys. Rev. A 55, 4556 (1997).

[59] O. Emile, M. Brunel, F. Bretenaker, and A. le Floch, Phys. Rev. A 57, 4889 (1998).

[60] H.-J. Sommers, Y. V. Fyodorov, and M. Titov, J. Phys. A 32, L77 (1999).

[61] Y. V. Fyodorov and B. Mehlig, Phys. Rev. E 66, 045202 (R).

[62] M. Born and E. Wolf, Principles of Optics (Cambridge University Press, New York, 1999).

[63] Corrections of the Fresnel laws due to the curvature of the interfaces are derived in M. Hentschel and H. Schomerus, Phys. Rev. E 65, 045603(R) (2002).

[64] T. Sh. Misirpashaev and C. W. J. Beenakker, Phys. Rev. A 57, 2041 (1998).

[65] For a recent discussion of thresholds for multimode lasing in absence of mode competition see O. Zaitsev, Phys. Rev. A 74, 063803 (2006).

[66] J. Jeffers, N. Imoto, and R. Loudon, Phys. Rev. A 47, 3346 (1993).

[67] C. W. J. Beenakker, Phys. Rev. Lett. 81, 1829 (1998).

[68] Equation (7) has been originally derived for paraxial resonators, where the wave function refers to the transverse profile of the modes [42, 43], but also applies to twodimensional dielectric resonators for which it can be derived directly from the Helmholtz equation.

[69] T. Harayama, S. Sunada, and K. S. Ikeda, Phys. Rev. A 72, 013803 (2005)

[70] E. Doron and U. Smilansky, Nonlinearity 5, 1055 (1992).

[71] B. Dietz and U. Smilansky, Chaos 3, 581 (1993).

[72] M. Hentschel and J. U. Nöckel, in Quantum Optics of Small Structures, edited by D. Lenstra, T. D. Visser, and H. A. H. van Leeuwen (Edita KNAW, Amsterdam, 2000).

[73] R. A. Janik, W. Nörenberg, M. A. Nowak, G. Papp, and I. Zahed, Phys. Rev. E 60, 2699 (1999).

[74] J. T. Chalker and B. Mehlig, Phys. Rev. Lett. 81, 3367 (1998).

[75] B. Mehlig and J. T. Chalker, J. Math. Phys. 41, 3233 (2000).

[76] B. Mehlig and M. Santer, Phys. Rev. E 63, 020105 (2001).

[77] F. Mezzadri, Notices Am. Math. Soc. 54, 592 (2007).

[78] H. Schomerus and J. Tworzydło, Phys. Rev. Lett. 93, 154102 (2004).

[79] J.P. Keating, M. Novaes, S. Prado and M. Sieber, Phys. Rev. Lett. 97, 150406 (2006).

[80] F. Haake, F. Izrailev, N. Lehmann, D. Saher, and H.-J. Sommers, Z. Phys. B 88, 359 (1992).

[81] N. Lehmann, D. Saher, V. V. Sokolov, and H.-J. Sommers, Nucl. Phys. A 582, 223 (1995).

[82] P. Sebbah, B. Hu, J. M. Klosner, and A. Z. Genack, Phys. Rev. Lett. 96, 183902 (2006). 